\title{
On the Performance of 1-Bit ADC in Massive MIMO Communication Systems
}

\author{
Hung N. Dang ${ }^{1}$, Thuy V. Nguyen ${ }^{1}$, Hieu T. Nguyen ${ }^{2}$ \\ 1 Faculty of Information Technology, Posts and Telecommunications Institute of Technology, Hanoi, \\ Vietnam \\ 2 Dept. of Science and Industry Systems, Faculty of Technology, Natural Sciences and Maritime Sciences, \\ University of Southeast Norway, NO 3603 Kongsberg, Norway
}

Correspondence: Thuy V. Nguyen, thuynv@ptit.edu.vn

Communication: received 13 July 2020, revised 5 August 2019, accepted 8 August 2020

Online publication: 21 August 2020, Digital Object Identifier: 10.21553/rev-jec.255

The associate editor coordinating the review of this article and recommending it for publication was Prof. Vo Nguyen Quoc Bao.

\begin{abstract}
Massive multiple-input multiple-output (MIMO) with low-resolution analog-to-digital converters is a rational solution to deal with hardware costs and accomplish optimal energy efficiency. In particular, utilizing 1-bit ADCs is one of the best choices for massive MIMO systems. This paper investigates the performance of the 1-bit ADC in the wireless coded communication systems where the robust channel coding, protograph low-density parity-check code (LDPC), is employed. The investigation reveals that the performance of the conventional 1-bit ADC with the truncation limit of 3-sigma is severely destroyed by the quantization distortion even when the number of antennas increases to 100. In particular, the optimized 1-bit ADC can achieved the iterative decoding threshold gain of $2 \mathrm{~dB}$ over the conventional 3-sigma 1-bit ADC at the coding rate of $1 / 2$ and the gain is more significant at higher coding rates. The optimized 1-bit ADC, though having substantial performance gain over the conventional one, is also affected by the quantization distortion at high coding rates and low MIMO configurations. Importantly, the investigation results suggest that the protograph LDPC codes should be re-designed to combat the negative effect of the quantization distortion of the 1-bit ADC.
\end{abstract}

Keywords- Protograph LDPC codes, large-scale MIMO, joint detection and decoding, PEXIT algorithm, 1-bit ADC.

\section{INTRODUCTION}

In future wireless networks such as $5 \mathrm{G}, 6 \mathrm{G}$, and beyond, the number of antennas at the order of tens and hundreds, so-called large-scale/massive multipleinput-multiple-output (massive MIMO) systems, is introduced as one of the vital technologies to attain high spectral efficiency, reliability, and power-saving [13]. Utilizing a large number of antennas in massive MIMO communication systems leads to some technical challenges to both the radio frequency (RF) module [4] and the baseband signal detection module [5]. Inside the radio frequency $(\mathrm{RF})$ module at the receiver side, multiple pairs of the analog-to-digital converter (ADC) and digital-to-analog converter (DAC) consume a large portion of the power and their hardware cost is high as well. The fact is that the hardware cost and the power consumption of ADCs and DACs grow linearly with the bandwidth and exponentially with the number of bits used in the ADCs, respectively. To tackle the difficulty, a potential solution is to replace the powerhungry high-resolution ADCs with low-power lowresolution ADCs [6-14].

In addition to the hardware impairment, the lowresolution $\mathrm{ADCs}$ lead to the error-floor behavior on the channel estimation error. Nonetheless, $\mathrm{Xu}$ et al., [11], verified via the uplink sum-rate that increasing the number of receive antennas at the base station (BS) can help to relieve the performance degradation caused by the low-resolution ADCs and the hardware impairment. Nguyen et al. developed learning techniques - exploiting the redundancy check or to-be-decoded data to aid the learning process - to deal with the circumstances where the channel state information (CSI) is imperfect or unavailable at the BS. The learning approach yields not only the performance improvement but also the robustness to the massive MIMO with low-resolution ADCs [10]. Using a similar strategy, Gao et al., [9], applied deep learning techniques to tackle the channel estimation problem, but for massive MIMO with the mixed ADCs (i.e., the system where a small portion of antennas has high-resolution ADCs while the rest has low-resolution ADCs). In this research, the approach to eliminating the adverse influence of the lowresolution ADCs is to utilize the signals received by the high-resolution ADC antennas to divine the channels of other antennas and their channels. This approach achieves performance improvement for the case of 1bit mixed ADCs.

Regarding the massive MIMO signal detection, the authors in [14] studied the two-stage signal detector based on the zero-forcing (ZF) and maximum likelihood (ML) detector for the massive MIMO systems with 1-bit ADCs. This introduced detector performs 
better than the conventional ZF detector, while its complexity is much lower than the ML detector. Dealing with 1-bit ADC MIMO systems where message-passing channel decoder is used, Cho et al. [13] developed an algorithm to compute the soft metric (e.g., a loglikelihood ratio) for the MIMO signal detector. The benefit of this approach is that the MIMO detector and the message-passing channel decoder can transfer the log-likelihood ratio of the coded bit with each other. The inter-stream interference is also canceled. As a result, this soft-output detector beats the ZFtype detector in both perfect and imperfect CSI cases at the BS. Besides, Nguyen et al., [12], investigate the coded massive MIMO systems where few-bit ADCs and protograph low-density parity-check (LDPC) codes are applied. The joint MIMO detection and decoding with parallel interference cancellation algorithm is used at the receiver. The investigation showed that a large number of antennas at the receiver could compensate for the low-resolution of the ADCs. Notably, the 4-bit ADC systems' performance can approach the performance of the high-resolution systems under various LS-MIMO configurations.

Though there is a good amount of research work on 1-bit ADCs for massive MIMO communication systems [9], [12], [14] and references therein, analyzing and optimizing its performance is limited, especially for the coded communication systems where the powerful lowdensity parity-check channel coding combined with the MIMO techniques is applied. The most relevant works to this research are from $\mathrm{Vu}$ et al. [12] and Dang et al. [15]. In [12], the joint belief propagation massive MIMO detection and protograph LDPC decoder was introduced coupled with the protographextrinsic information exchange chart (PEXIT), which is used to attain the analytical evaluation of a given channel code and to design new protograph LDPC codes for LS-MIMO fading channels. However, there are two limits in this work: 1) The work investigated the resolution limited from 2-bit to 5-bit, 2) the truncation limited is fixed by using the 3-sigma rule. As proved in [15], the 3-sigma rule is good for highresolution ADCs (from 3 bits or higher). For lowresolution ADCs, especially 1-bit ADCs, the ADCs with 3-sigma rule have performance loss of up to 9 bits/s/Hz in comparison with the optimized one. Dang et al. optimized the truncation limits for the uniform scalar quantizer according to the resolution levels of the ADCs in order to minimize the overall quantization distortion. Ultimately, the achievable sum-rate of uplink multiuser MIMO (MU-MIMO) systems is significantly improved for the practical resolution level of the ADCs, especially for an extreme case of 1-bit ADCs. The results show that the optimized uniform scalar quantizer, on the one hand, can improve the sum-rate as much as $9 \mathrm{bits} / \mathrm{s} / \mathrm{Hz}$ and 2 bits/s/Hz for 1-bit ADCs and 2-bit $\mathrm{ADCs}$, respectively. On the other hand, the performance of the optimized uniform quantizer approaches the performance of the non-uniform quantizer at all considered resolution levels of the ADCs. Unfortunately, this research results are obtained by using the information- theoretical approach (i.e., assuming that the random channel coding with infinite information blocklength is used in the massive MIMO coded communication systems).

Motivated by the above observation, this paper investigates the performance of 1-bit ADCs for massive MIMO coded communication systems where pragmatic protograph codes are applied. The performance analysis will provide the practical performance gap of the optimized and the 3-sigma uniform scalar quantizers. In particular, both the iterative decoding threshold of protograph codes and the bit error rate (BER) are two useful metrics that we use to analyze massive MIMOcoded communication systems. The main contributions of the paper are summarized below:

- The paper proposes a wireless coded communication scheme where powerful and low-complexity protograph LDPC codes combined with the MIMO transmission scheme are employed. The truncation limit is calculated for the massive MIMO channel under the assumption that the received signal at each receiving antenna follows the Gaussian distribution.

- The iterative decoding thresholds are calculated for various MIMO configurations using the new protograph LDPC codes designed for large-scale MIMO channels. The extensive BER curves are produced to verify the analytical results. Both simulation and analytical results indicate that the performance of the 1-bit ADC with the 3-sigma rule is severely destroyed by the quantization distortion even though the 3-sigma rule works for the higher resolution from 2-bit to 5-bit ADCs.

- The investigation in this research reveals that the optimized 1-bit ADC, though having better performance than the 3-sigma one, is also affected by the quantization distortion at the low MIMO configurations and high coding rates. Our study shows that a large number of antennas play an essential role in combating the negative effect of the distortion noise of 1-bit ADC. Observing the system's performance at high coding rates suggests that the new protograph LDPC codes should be redesigned/re-optimized for the particular case of 1bit ADC to achieve better system performance.

The paper is organized as follows. Section 2 presents the description of the massive MIMO channel model and the 1-bit quantization modeling as well as the full details of the joint belief propagation massive MIMO detection and protograph LDPC decoder, which is proven the good solution to reduce the complexity of the receiver hardware. The performance evaluation of 1-bit ADCs is detailed in Section 4 in various scenarios. Here, the new protograph LDPC codes designed for massive MIMO channel, [16], are chosen to perform the analysis. Both the iterative decoding threshold and the BER performance of the protograph LDPC codes are included. Section 5 concludes the paper. 


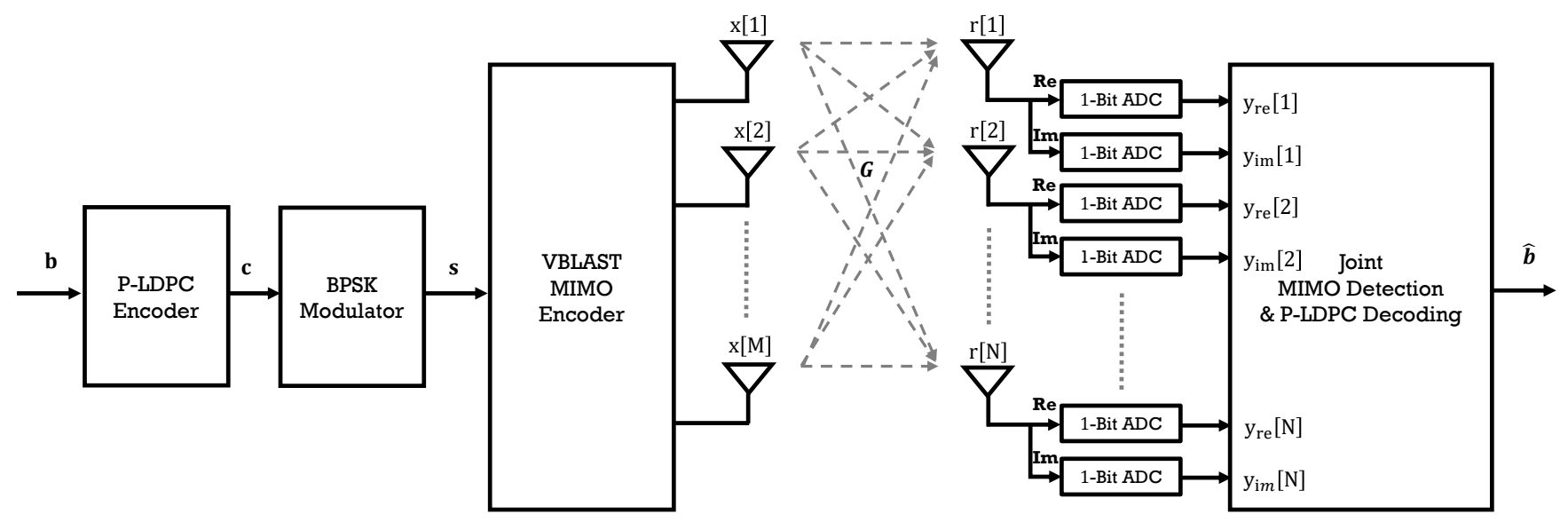

Figure 1. The channel model of the LS-MIMO coded communication with 1-bit ADC where Vertically-layered Bell Laboratories Layered SpaceTime (V-BLAST) technique is used.

\section{System Model}

Consider a wireless coded communication system whose wireless fading multiple-input-multiple-output (MIMO) channel has $M$ inputs (transmitting antennas) and $N$ output (receiving antennas). In addition, 1-bit ADCs are employed at the receiver side as shown in Figure 1.

In particular, a block of $L_{i}$ information bits is first encoded by a P-LPDC encoder that produces a codeword with a length of $L_{c}$ coded bits. The value of $L_{c}$ relates to the value of $L_{i}$ via the coding rate $R=L_{i} / L_{c}$. The coded bits $c \in\{0,1\}$ are modulated by a binary-phase-shiftkeying (BPSK) modulator whose output levels belongs to the set $s=(-1)^{c} \in\{+1,-1\}$. In one channel use, using the spatial multiplexing scheme [17], $M$ modulated symbols are transmitted over $M$ transmitting antennas. It thus requires $L=\left\lceil L_{c} / M\right\rceil$ channel uses to transfer all $L_{c}$ coded bits.

Such massive MIMO channel is mathematically modeled as

$$
\mathbf{r}=\mathbf{G} \mathbf{x}+\mathbf{w},
$$

where $\mathbf{x}=[x[1], x[2], \ldots, x[M]]^{T}$ is the transmitted MIMO symbol whose elements belong to the BPSK modulation alphabet. The average symbol energy $E_{s}=$ $\mathbb{E}\left(\|\mathbf{x}\|^{2}\right)$ is normalized to $1 . \mathbf{G} \in \mathbb{C}^{N \times M}$ is channel gain matrix whose entries $g[n, m]$ in the $n$-th row and $m$-th column of $\mathbf{G}$ are assumed to be independent and identical distribution (i.i.d.) complex Gaussian with zero mean and unit variance $\mathcal{C N}(0,1)$. In this paper, we assume that the perfect channel state information (CSI) is available at the receiver, but not at the transmitter. The noise vector $\mathbf{w}=[w[1], w[2], \ldots, w[N]]^{T} \in \mathbb{C}^{N \times 1}$ is assumed to be complex additive white Gaussian noise vector whose entries follow i.i.d. complex Gaussian with zero mean and $N_{0}$ variance (i.e., $\mathcal{C} \mathcal{N}\left(0, N_{0}\right)$ ). Finally, $\mathbf{r}=[r[1], r[2], \ldots, r[N]]^{T} \in \mathbb{C}^{N \times 1}$ is the received signal vector whose element $r[n]$ is the received signal at the $n$-th antenna.

The received signal at each receiving antenna, $r[n]$, $n=1,2, \ldots, N$, is first transformed from the analog form to the digital form by a pair of 1-bit ADCs at the RF module: One 1-bit ADC is for the in-phase (real) signal and the other 1-bit ADC is for the quadrature (imaginary) signal. The quantized version of the received signal is finally fed to the joint MIMO detection and protograph LDPC decoding algorithm [12] to restore the original information bits. In the following two subsections, we will present in detail the 1-bit quantization and the joint MIMO detection and protograph LDPC decoding algorithm before proceeding to perform the performance analysis.

\section{Performance Analysis}

\subsection{1-Bit Quantization Modeling}

Let $Q$ be the quantization operator, the relation between the input and output of the 1-bit ADC block is given by

$$
\mathbf{y}=\mathbb{Q}\left(\mathbf{r}_{r e}\right)+j \mathbb{Q}\left(\mathbf{r}_{i m}\right),
$$

where $\mathbf{r}_{r e}$ and $\mathbf{r}_{i m}$ are the real and imaginary parts of the received signal $\mathbf{r}$, respectively. Moreover, the quantizer $Q$ is the scalar and uniform one (i.e., each component in the vector is quantized independently, and the quantization intervals have equal length).

In this work, we employ the additive quantization noise model (AQNM) in MIMO systems with lowresolution ADCs $[4,18]$ to optimize the 1-bit ADCs. Here, the quantization noise is modeled as the noise component which is added to the input signal. The relationship between the input and output of the quantizer in (2) is particularly expressed as below [4]

$$
\mathbf{y}=\varphi \mathbf{r}+\mathbf{w}_{Q},
$$

where $\varphi=1-\rho$, which is the performance metric of a given quantizer, and $\rho$ is the inverse of the signalto-quantization-distortion ratio and $\mathbf{w}_{Q}$ is the additive quantization noise.

As shown later, the larger the value of $\varphi$, the better the performance of the massive MIMO coded communication system. Therefore, to improve the performance 
Table I

Optimal Truncation Limits for Uniform AND Scalar QUANTIZER of THE 1-BIT ADC

\begin{tabular}{|c|c|c|c|}
\hline & 3-Sigma Uniform Quant. & Optimized Uniform Quant. & Non-uniform Quant. \\
\hline$T_{N_{Q}}$ & 3 & 1.669 & Not Available \\
\hline$\varphi$ & 0.1371 & 0.6261 & 0.6366 \\
\hline
\end{tabular}

of a given massive MIMO coded communication system, one should optimize to achieve a higher value of $\varphi$ for a given 1-bit ADC by reducing the quantization noise of the quantizer. In this paper, we show that by optimizing the quantizer's truncation limit, the quantization will dramatically be reduced. This is the design goal of this subsection.

Observing from the channel model in (1), the input signals of the 1-bit ADCs have the probability density functions (pdf) following the normal distribution by the light of the law of large number argument. Therefore, they are continuous random variables with infinite support. Accordingly, the input signal, $r[n]$, is first normalized with its standard deviation to have unit variance. Next, the normalized version of the received signal $r^{*}[n], n=1,2, \ldots, N$ is truncated to ensure that the input signal of the uniform scalar quantizer has the finite support in the range $\left[-T_{N_{Q}}, T_{N_{Q}}\right]$ as below [15]:

$$
r_{T}[n]= \begin{cases}-T_{N_{Q^{\prime}}} & r^{*}[n]<-T_{N_{Q^{\prime}}} \\ r^{*}[n], & -T_{N_{Q}} \leq r^{*}[n] \leq T_{N_{Q^{\prime}}} \\ T_{N_{Q^{\prime}}} & r^{*}[n]>T_{N_{Q^{\prime}}}\end{cases}
$$

where $r^{*}[n]$ is the normalized version of $r[n]$ and $r_{T}[n]$ be the truncated version of the received signal $r^{*}[n]$ and $N_{Q}=2$ is the number of intervals.

The truncated signal is then supplied to the uniform scalar quantizer whose step size $\Delta=2 T_{N_{Q}} / N_{Q}$. As explained, the quantization follows two processes: 1) the truncation process to limit the range of the input signal; 2) representing/mapping process (i.e., assign a suitable representation/middle point to the input signal) to convert the signal with infinite levels into finite levels. The truncating process produces the overload distortion, which is dependent on the value of $T_{N_{Q}}$ and the pdf of the input signal. In contrast, the representing process causes granular distortion, which is dependent on the number of quantization levels, $N_{Q},[19]$.

Many previous research works of quantized massive MIMO systems use the 3-sigma rule, for example, in [20], to determine the truncation, i.e., $T_{N_{Q}}=3$, regardless of the resolution of the ADCs. As proved in [15], the quantizer with the 3-sigma rule possesses inferior performance merit compared with the non-uniform quantizer in [4], especially for the lowresolution of the ADCs. The reason for the 3-sigma rule quantizer's poor performance is that the overload distortion and the granular distortion are not wellbalanced. More specifically, the granular distortion is much more than the overload distortion at the low resolution.

To reach the design goal mentioned earlier - reducing the quantization, the value of $T_{N_{Q}}$ for the 1-bit ADCs is optimized to achieve the optimal balance between the overload distortion and the granular distortion. Once the number of quantized intervals, $N_{Q}=2$, is fixed, the optimal value of $T_{N_{Q}}$ depends on the probability density function of the input signal. Providentially, since the received signal is assumed to follow the normal distribution, we can apply the result derived by Hui et al. [21] to find the optimal truncation limit to minimize the overall quantization noise. For the normal distribution signal, the optimal truncation limit $T_{N_{Q}}$ is two-side bounded as [21]

$$
F^{-1}\left(\frac{1+\delta}{6 N_{Q}}\right)<T_{N_{Q}}<F^{-1}\left(\frac{1-\delta}{6 N_{Q}^{2}}\right),
$$

where the $F^{-1}()$ is the inverse function of the following function

$$
F(y)=\frac{1}{\sqrt{2 \pi}} \frac{1}{y} \exp \left(-\frac{y^{2}}{2}\right)
$$

Denote

$$
T_{N_{Q}}^{l}=F^{-1}\left(\frac{1+\delta}{6 N_{Q}}\right)
$$

and

$$
T_{N_{Q}}^{u}=F^{-1}\left(\frac{1-\delta}{6 N_{Q}^{2}}\right)
$$

For each value pair of $\delta$ and $N_{Q}$, we solve Equations (7) and (8) to find corresponding values of $T_{N_{Q}}^{l}$ and $T_{N_{Q^{\prime}}}^{u}$ respectively. Finally, we choose the optimal value to $T_{N_{O}}$ is the mid-point of the upper bound and the low bound in (5) as follows

$$
T_{N_{Q}}^{*}=\frac{1}{2}\left(T_{N_{Q}}^{l}+T_{N_{Q}}^{u}\right) .
$$

Table I provides the optimal value of $T_{N_{Q}}$ for the 1-bit ADCs and the corresponding $\varphi$ values. We see that the optimal truncation limit of the 1-bit ADC is 1.669 , which is far less than the value of 3-sigma rule 1-bit ADC. The optimal value of $T_{N_{Q}}$ should be small to achieve a good balance between the overloaded distortion and granular distortion. Correspondingly, the value of quantizer performance metric $\varphi$ is thus much more improved for the optimized 1-bit quantizer. More specifically, at 1-bit ADC or only two quantization levels, the value $\varphi$ of the 3 -sigma quantizer is 0.1371 , whereas the value $\varphi$ of the optimized quantizer is 0.6261 . This is a significant performance gap, and we should expect that the performance of the optimized 1-bit ADC will be far better than that of the 3-sigma 1-bit ADC. This claim will be verified in the later section via both the iterative decoding threshold and the BER performance under the various experiment of the MIMO configurations and code rates.

One impressive result is that the optimized 1-bit ADC performance is very close to the non-uniform quantizer 
where the interval levels are optimized according to the pdf of the input. The difference in $\varphi$ value is of 0.01 a marginal gap.

\subsection{Joint Double-Layer Belief Propagation Receiver}

The joint double-layer belief propagation receiver, $[12,16]$, is an excellent solution for the massive MIMO coded communication systems because of two reasons:

1) The complexity of the receiver is low.

2) The performance is remarkable since the extrinsic information is transferred between the MIMO detector layer and the protograph LDPC code layer. Hence, we use this receiver architecture in this research to analyze the performance of the 1-bit ADC. For the sake of completeness, the joint double-layer belief propagation receiver will be briefly presented below.

When the number of antennas is significant, in order of tens or hundreds, the traditional MIMO detection algorithms such as zero-forcing, minimum mean square error spatial filtering, sphere decoding, and maximum likelihood detector are computationally restrictive [5, 22]. Alternatively, the message-passing algorithm is a promising solution to deal with the complexity issue. Nguyen et al. [12] and $\mathrm{Vu}$ et al. [16] introduced the joint belief propagation decoder for large-scale MIMO coded communication systems where $\mathrm{Vu}$ et al. [16] used the double-layer graph to search for good protograph LDPC codes for the LS-MIMO channel and Nguyen et al. [12] employed the joint double-layer belief propagation receiver to analyze the performance of low-resolution ADCs (from 2 bit to 5 bit).

Those are two research works that are closed the topic of this paper. But, neither of them deals with the performance of the coded communication systems with 1-bit ADCs. This paper will fill the gap to investigate and present the performance of 1-bit ADCs with the double-layer belief propagation receiver. The results will provide the engineering insights of how to apply and design the wireless massive MIMO coded communication systems with the 1-bit ADCs.

To explain the joint detection and decoding algorithm, we utilize a double-layer graph, as shown in Figure 2. The double-layer graph has three types of nodes, namely:

1) $L \times N$ observation nodes are representing the received signal sequence $\mathbf{y}$.

2) $L_{c}=L \times M$ symbol nodes that represent the transmit symbol sequence $\mathbf{x}$.

3) Finally, there are $K=L_{c}-L_{i}$ check nodes representing the check equations of given P-LDPC codes.

The connection of the variable node and the check node is ruled by the parity matrix of the LDPC code. In one channel use, the $N$ observation nodes and the $M$ symbol nodes are fully linked to create a graph for the MIMO detection part (i.e., one observation node is linked to all $M$ symbol nodes). In the graph for the LDPC decoding part, there are $L_{c}$ variable nodes representing the codeword bit sequence $c$. With the BPSK modulation scheme, the one-one mapping is

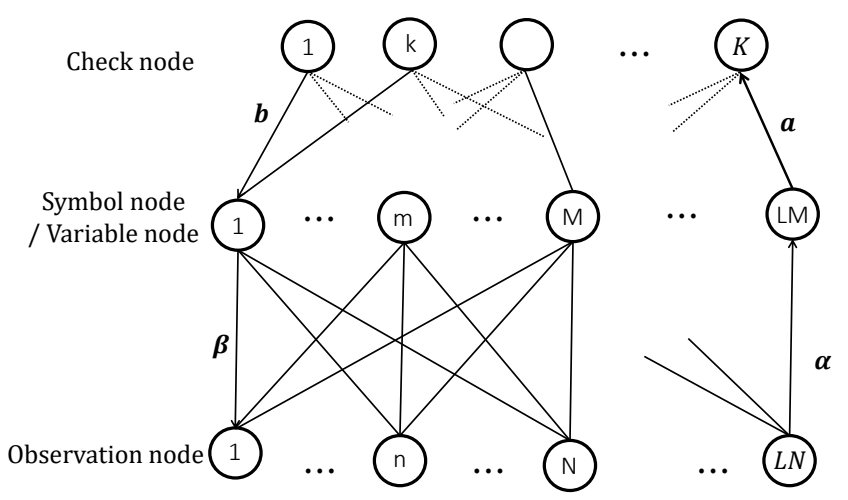

Figure 2. Joint double-layer belief propagation receiver.

used to map a codeword bit to a transmitting symbol. Consequently, the variable node and the symbol node are joined in a single node on the double-layer graph. Hence, the two terms, the variable node and symbol node, are used interchangeably in this paper.

In the iterative joint detection and decoding algorithm on the double-layer graph, there are five types of messages passed over the graph as follows:

- $\alpha[n, m]$ is the message passed from the $n$-th observation node to the $m$-th symbol node.

- $a[m, k]$ is the message passed from the $m$-th variable node to the $k$-th check node.

- $b[k, m]$ is the message passed from the $k$-th check node to the $m$-th variable node.

- $\beta[m, n]$ is the message passing from the $m$-th symbol node to the $n$-th observation node.

- $\Gamma[m]$ is the a posteriori log-likelihood ratio (LLR) value of the symbol $x[m]$.

In the sequel, we briefly explain the working principle of the message passing joint detection and decoding receiver with soft symbol cancellation. Further details can be found in $[12,16,23]$.

3.2.1 Message Passed From Observation Nodes To Symbol Nodes: The received signal at the $n$-th observation node is given as

$$
\begin{aligned}
y[n, m]= & \varphi r[n]+w_{Q}[n] \\
= & \varphi \sum_{m=1}^{M} g[n, m] x[m]+\varphi w[n]+w_{Q}[n] \\
= & \varphi g[n, m] x[m]+\varphi \underbrace{\varphi \sum_{t=1, t \neq m}^{M} g[n, t] x[t]}_{\text {Interference }} \\
& +\varphi w[n]+w_{Q}[n] .
\end{aligned}
$$

We can now rewrite $\hat{y}[n, m]$ as below

$$
\hat{y}[n, m]=\varphi g[n, m] x[m]+z[n, m],
$$

with

$$
\begin{aligned}
z[n, m]= & \varphi \sum_{t=1, t \neq m}^{M} g[n, t](x[n, t]-\hat{x}[n, t]) \\
& +\varphi w[n]+w_{Q}[n] .
\end{aligned}
$$

The message passed from the $n$-th observation node to the $m$-th variable node is the log-likelihood ratio 
(LLR) and given by

$$
\begin{aligned}
\alpha[n, m] & =\ln \frac{\operatorname{Pr}(\hat{y}[n, m] \mid \mathbf{G}, x[m]=+1)}{\operatorname{Pr}(\hat{y}[n, m] \mid \mathbf{G}, x[m]=-1)} \\
& =\frac{4 \varphi}{\Psi[n, m]} \mathfrak{R}\left(g^{*}[n, m] \hat{y}[n, m]\right),
\end{aligned}
$$

where

$$
\begin{aligned}
\Psi[n, m]= & \varphi^{2} \sum_{t=1, t \neq m}^{M}|g[n, t]|^{2}\left(1-|\hat{x}[n, t]|^{2}\right) \\
& +\varphi^{2} N_{0}+\varphi(1-\varphi)\left(\sum_{m=1}^{M}|g[n, m]|^{2}+N_{0}\right) .
\end{aligned}
$$

There are total $N$ messages sent to a given symbol node (or transmit symbol), and the sum of all the messages is equivalent to the channel message (i.e., $L_{c h}$ ) in the conventional message-passing algorithm [24]. Compared to the expression derived in [22], the new expression in (13) takes into account the quantization noise effect via the parameter $\varphi$ and $\Psi[n, m]$, which depend on the performance metric of the 1-bit ADCs and the fading channels as aforementioned.

When the 3-sigma 1-bit ADCs is used, the factor $4 \varphi / \Psi[n, m]$ in (13) decreases as $\varphi$ of the three-sigma 1 -bit ADCs is very poor as shown in Table I. The channel message, $\alpha[n, m]$ sent to the variable nodes in (13) therefore decreases. Ultimately, the performance of the joint double-layer belief propagation receiver is degraded accordingly.

3.2.2 Message Passed From Variable Nodes To Check Nodes: Considering the $m$-th variable node, two types of messages are sent to this node. The first type of messages is from the $N$ observation nodes belonging to the part of the MIMO detection graph, and the other type of messages is from the check nodes belonging to the part of the LDPC decoding graph. As a result, the extrinsic message from the $m$-th variable node to the $k$-th check node is the sum of all the messages from the observation nodes and the check nodes except the message from the $k$-th check node. We have

$$
a[m, k]=\sum_{t \in \mathcal{N}_{0}(m)} \alpha[t, m]+\sum_{t \in \mathcal{N}_{c}(m) \backslash k} b[t, m],
$$

where $\mathcal{N}_{c}(m)$ is the set of check nodes connected to the $m$-th variable node, and $\mathcal{N}_{o}(m)$ is the set of observation nodes connected to the $m$-th variable node.

3.2.3 Message Passed From Check Nodes to Variable Nodes: The message from the $k$-th check node to the $m$-th variable node is identical to the conventional message-passing algorithm [24] and given by

$$
b[k, m]=\ln \frac{1-\prod_{t \in \mathcal{N}_{v}(k) \backslash m} \frac{1-e^{a[t, k]}}{1+e^{a[t, k]}}}{1+\prod_{t \in \mathcal{N}_{v}(k) \backslash m} \frac{1-e^{a[t, k]}}{1+e^{a[t, k]}}},
$$

where $\mathcal{N}_{v}(k)$ is the set of variable nodes connected to the $k$-th check node. In practical implementation, the computation of $b[k, m]$ is simplified by using the $\tanh (\cdot)$ function.
3.2.4 Message Passed From Symbol Nodes To Observation Nodes: As mentioned above, the $m$-th symbol node receives messages from both the observation nodes and the check nodes. The extrinsic message sent from the $m$-th symbol node to the $n$-th observation node is the sum of all the messages except the message from the $n$-th observation node. As a result, the message from the $m$-th variable node to the $n$-th observation node is given by

$$
\beta[m, n]=\sum_{t \in \mathcal{N}_{o}(m) \backslash n} \alpha[t, m]+\sum_{t \in \mathcal{N}_{c}(m)} b[t, m],
$$

where $\mathcal{N}_{o}(m)$ and $\mathcal{N}_{c}(m)$ are the sets of all observation nodes and check nodes that are connected to the $m$-th symbol node, respectively.

3.2.5 A posteriori messages of codeword bits: The posterior LLR of the $m$-th transmit symbol at the end of each iteration is the total messages from both the observation nodes and the check nodes, and it is given by

$$
\Gamma[m]=\sum_{n \in \mathcal{N}_{0}(m)} \alpha[n, m]+\sum_{k \in \mathcal{N}_{c}(m)} b[k, m] .
$$

The posteriori LLR is sent to the hard decision device to produce the decoded version of the codeword bit using the following rule:

$$
\hat{c}[m]=\left\{\begin{array}{l}
0, \Gamma[m]>0, \\
1, \text { Otherwise, }
\end{array}\right.
$$

where $\hat{c}[m]$ denotes the decoded version of $c[m]$. And thus, the decoded sequence of the information $\hat{\mathbf{b}}$ is obtained.

The message-passing process stops when all check equations are satisfied, or the maximum number of iterations is reached. Otherwise, the message-passing process repeats with a message update from the observation nodes.

\section{Numerical Results}

\subsection{Iterative Decoding Threshold}

In this section, we use the large-scale MIMO PEXIT (LS-MIMO-PEXIT) algorithm, previously proposed in $[12,16]$, to evaluate the performance improvement of the optimized 1-bit uniform quantizer. Since utilizing the LS-MIMO PEXIT for 1-bit ADCs is straightforward, readers refer to those two references for more details.

The LS-MIMO-PEXIT was proved to be a useful tool for evaluating and designing protograph LDPC codes through the iterative decoding threshold. This is the lowest received signal-to-noise ratio that the decoder can decode the noisy bitstream. Thus, the lower the iterative decoding threshold, the better the communication systems can achieve.

To calculate the iterative decoding threshold, we select the protograph LDPC codes that were previously optimized for LS-MIMO channels and joint doublelayer belief propagation receiver [16]. The selected protograph LDPC codes are given in (20), (21), (22). 


$$
\begin{aligned}
\mathbf{B}_{1 / 2}^{\text {20iter. }} & =\left(\begin{array}{llllll}
3 & 1 & 1 & 0 & 0 & 1 \\
2 & 1 & 2 & 2 & 1 & 0 \\
3 & 2 & 0 & 1 & 1 & 0
\end{array}\right)_{3 \times 6}, \\
\mathbf{B}_{2 / 3}^{20 \text { iter. }} & =\left(\begin{array}{lll|l}
3 & 0 & 0 & \\
2 & 3 & 0 & \mathbf{B}_{1 / 2}^{20 \text { iter. }} \\
3 & 0 & 2 &
\end{array}\right)_{3 \times 9}, \\
\mathbf{B}_{3 / 4}^{\text {20iter. }} & =\left(\begin{array}{lll|l}
3 & 0 & 0 \\
2 & 2 & 2 \\
1 & 1 & 1 & \mathbf{B}_{2 / 3}^{20 i t e r .}
\end{array}\right)_{3 \times 12} .
\end{aligned}
$$

Table II

Iterative Decoding Threshold: Code Rate 1/2

\begin{tabular}{|c|c|c|}
\hline MIMO Configuration & 3-Sigma & Optimized \\
\hline $10 \times 10$ & 5.47 & 3.07 \\
\hline $40 \times 40$ & 5.03 & 2.84 \\
\hline $100 \times 100$ & 5.21 & 2.97 \\
\hline
\end{tabular}

Table III

Iterative Decoding Threshold: Code Rate 2/3

\begin{tabular}{|c|c|c|}
\hline MIMO Configuration & 3-Sigma & Optimized \\
\hline $10 \times 10$ & 14.09 & 4.89 \\
\hline $40 \times 40$ & 11.52 & 4.39 \\
\hline $100 \times 100$ & 11.71 & 4.42 \\
\hline
\end{tabular}

Table IV

Iterative Decoding Threshold: Code Rate 3/4.

\begin{tabular}{|c|c|c|}
\hline MIMO Configuration & 3-Sigma & Optimized \\
\hline $10 \times 10$ & 15.99 & 6.46 \\
\hline $40 \times 40$ & 15.99 & 5.71 \\
\hline $100 \times 100$ & 15.99 & 5.67 \\
\hline
\end{tabular}

The iterative decoding thresholds for coding rates $1 / 2,2 / 3$, and $3 / 4$ are given in Table II, Table III, and Table IV, respectively. As expected, the iterative decoding thresholds for the optimized quantizer are significantly lower than the 3-sigma (conventional) quantizer in all MIMO configurations. The threshold gaps vary from 2 $\mathrm{dB}$ (at coding rate $1 / 2$ ) to $10 \mathrm{~dB}$ (at coding rate $3 / 4$ ). Therefore, one should expect huge gaps between the BER curves of the two quantization schemes. To be specific, the optimized 1-bit ADC will have much highperformance merit or very low BER at the same level of signal to noise ratio (SNR). This analytical finding will be verified by simulation results in the below subsection.

\subsection{Bit Error Rate Performance}

In this section, the simulation results, as shown in Figures 3-11, are provided to verify the analytical results in Subsection 4.1. It is immediately seen that the BER curves of the optimized quantizer are far lower than those of the 3-sigma quantizer for the whole considered range of SNR. This phenomenon is in good agreement with the finding from the analytical results.

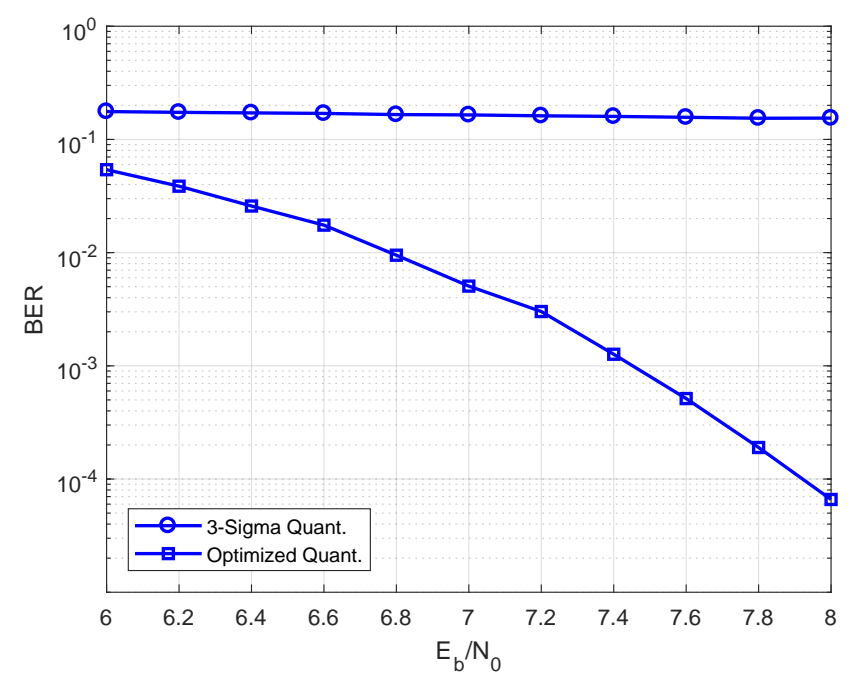

Figure 3. BER performance: $10 \times 10$ MIMO Configuration, Coding rate $R=1 / 2$, Coded blocklength $=2400$ bits.

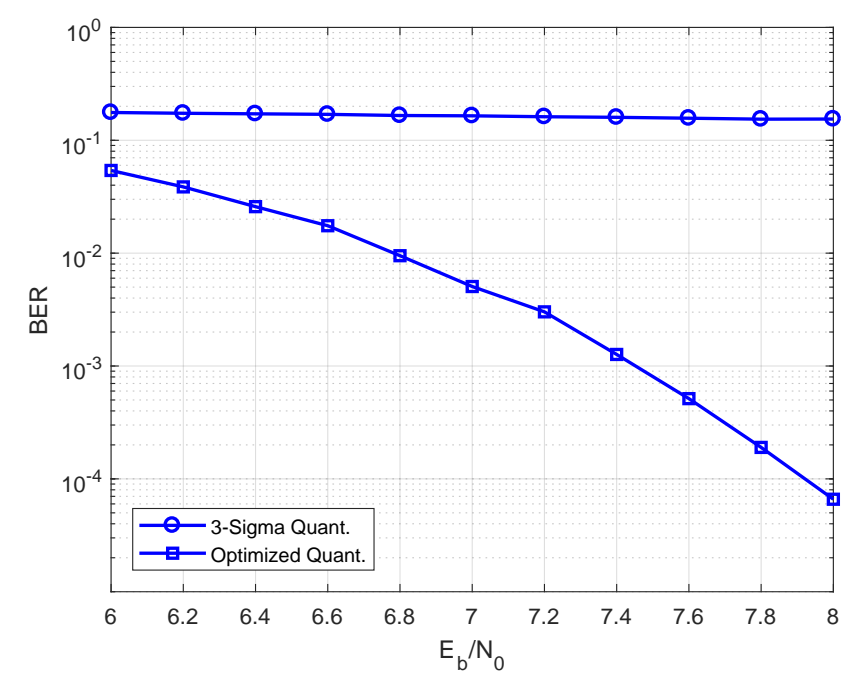

Figure 4. BER performance: $40 \times 40$ MIMO Configuration, Coding rate $R=1 / 2$, Coded blocklength $=2400$ bits.

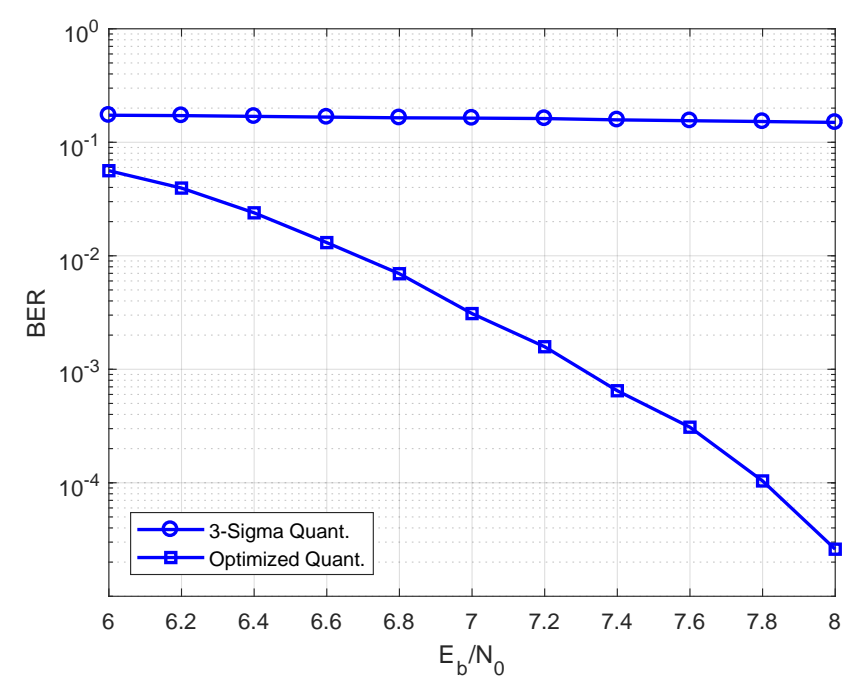

Figure 5. BER performance: $100 \times 100$ MIMO Configuration, Coding rate $R=1 / 2$, Coded blocklength $=2400$ bits. 


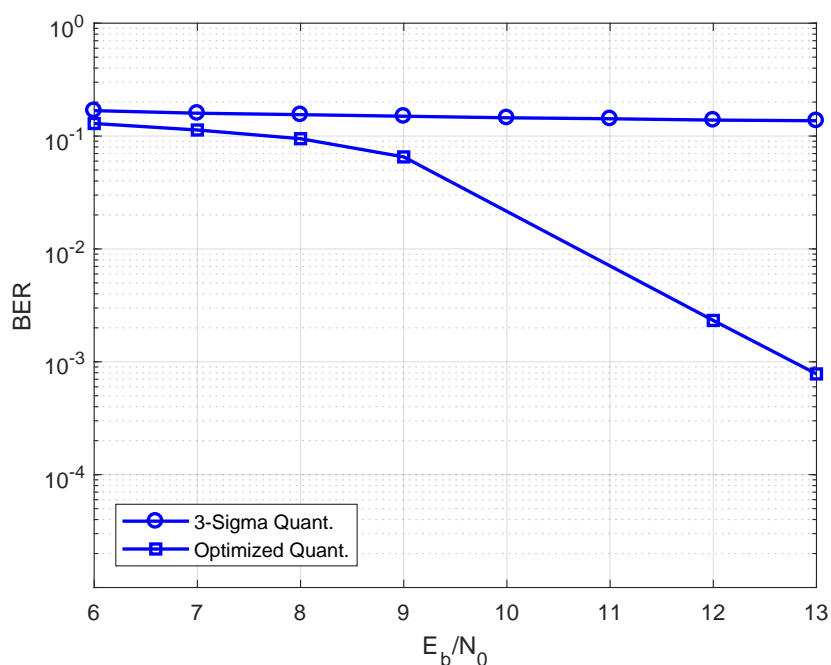

Figure 6. BER performance: $10 \times 10$ MIMO Configuration, Coding rate $R=2 / 3$, Coded blocklength $=2400$ bits.

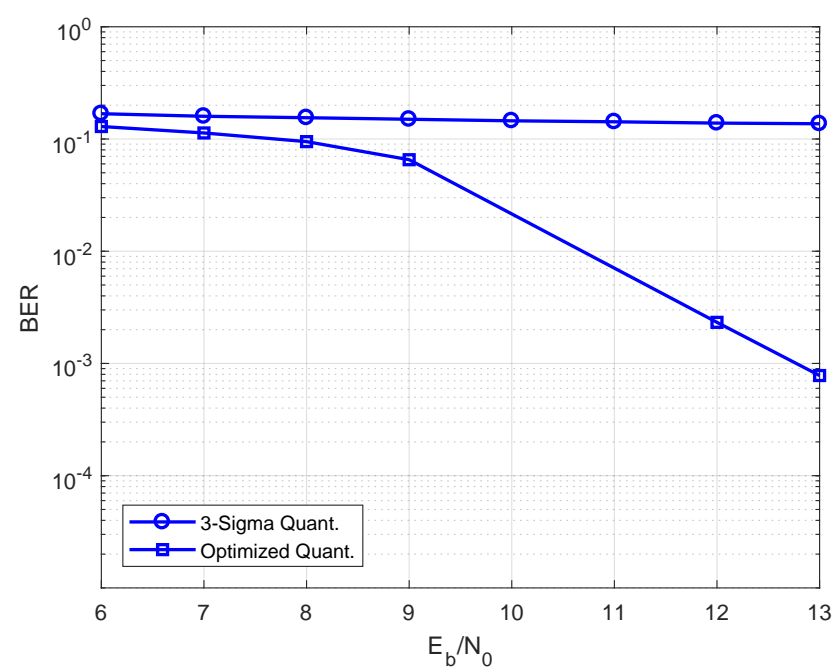

Figure 7. BER performance: $40 \times 40$ MIMO Configuration, Coding rate $R=2 / 3$, Coded blocklength $=2400$ bits.

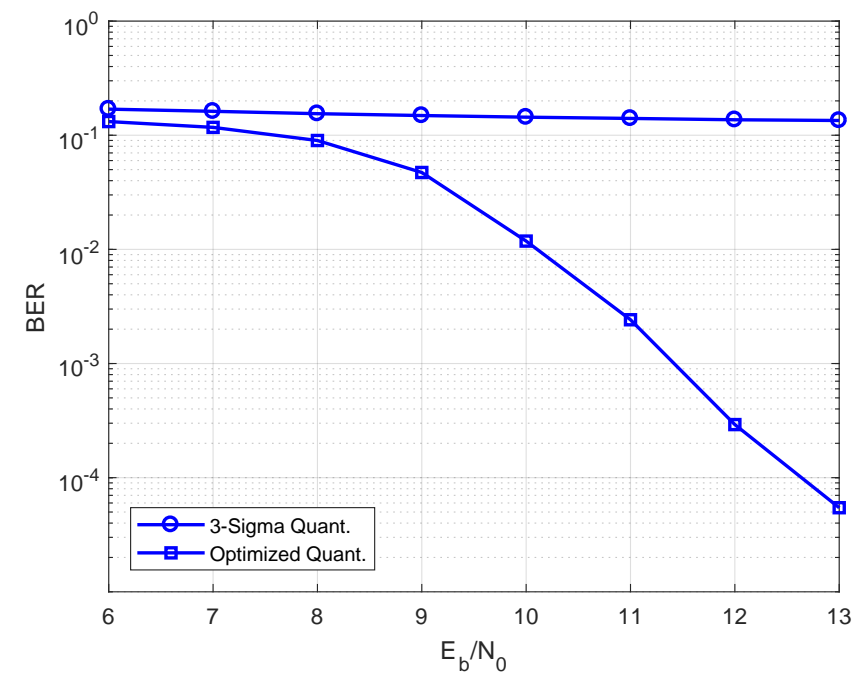

Figure 8. BER performance: $100 \times 100$ MIMO Configuration, Coding rate $R=2 / 3$, Coded blocklength $=2400$ bits.

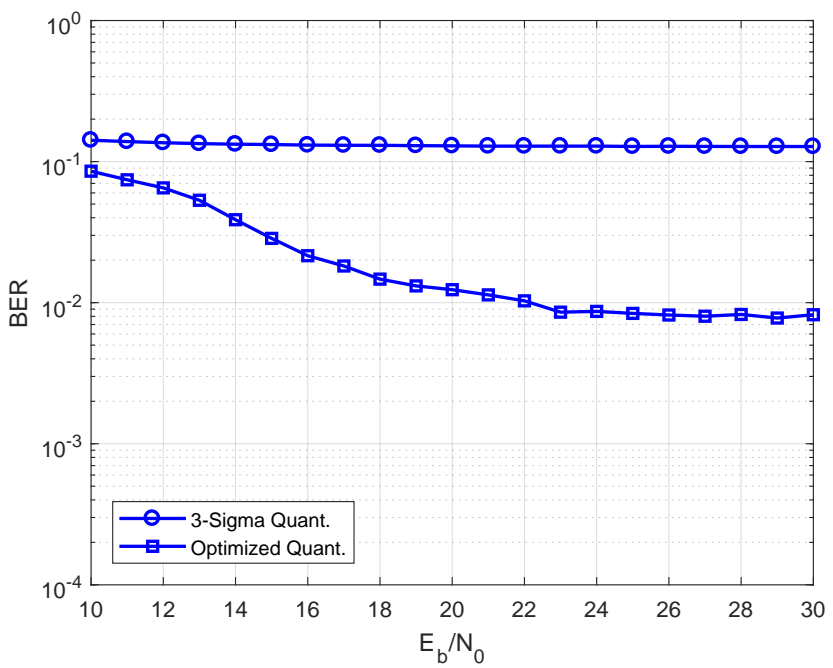

Figure 9. BER performance: $10 \times 10$ MIMO Configuration, Coding rate $R=3 / 4$, Coded blocklength $=2400$ bits.

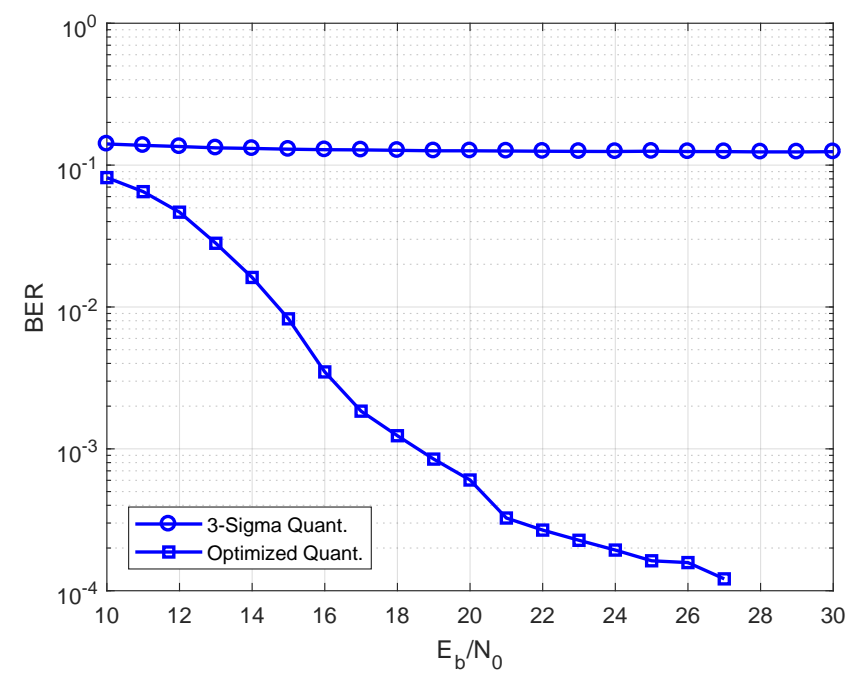

Figure 10. BER performance: $40 \times 40$ MIMO Configuration, Coding rate $R=3 / 4$, Coded blocklength $=2400$ bits.

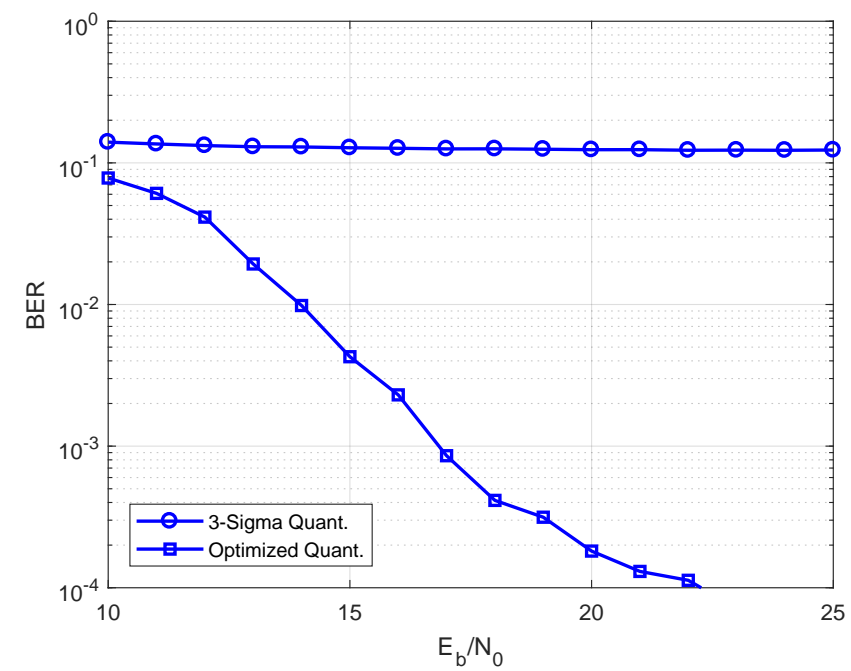

Figure 11. BER performance: $100 \times 100$ MIMO Configuration, Coding rate $R=3 / 4$, Coded blocklength $=2400$ bits. 
Note that the BER gaps are not the same as the iterative decoding threshold gaps since the LS-MIMO-PEXIT is designed using an approximation method $[12,16]$. Therefore, the iterative decoding threshold only helps to indicate the behavior tendency, not the absolute metric to evaluate the performance.

The 3-sigma quantizer has poor performance in all coding rates and MIMO configurations. Even the number of transmitting and receiving antennas is in the order of 100, the BER performance of the 3-sigma quantizer is still floored. This observation means that the truncation limit is especially important for 1-ADCs. This fact was proved from the information-theoretical work in [15]. Nevertheless, for higher resolution (i.e., from 2-bit ADCs to 5-bit ADCs), the 3-sigma is still converged in both coded [12] and uncoded communication systems [23].

Look at the performance of the optimized quantizer alone, the slopes of the BER curves at the coding rate of $1 / 2$ are steepest, and the BER performance is smooth and stable in all the MIMO configurations. Nevertheless, at the coding rate of $2 / 3$, the performance is significantly degraded when the MIMO configuration is $10 \times 10$. The performance is extremely destroyed at a higher coding rate $(R=3 / 4)$. In particular, the BER curve is floored at BER $=10^{-2}$ with the $10 \times 10$ MIMO configuration. When the number of antennas increases to 40 or 100, the BER curves indicate the performance improvement. The observing results imply that 1-ADCs should be used in the massive MIMO so that the negative effect of the quantization distortion is relieved by a massive number of receiving antennas. This finding was found via the information-theoretical analysis for uplink MIMO multiuser scenarios [11].

The experiment results in this research also suggest that the protograph LDPC codes should be re-designed to combat the effect of the quantization distortion for the high coding rate, for example, $R=2 / 3$, and $R=$ $3 / 4$. The code design framework will be presented in another report.

\section{Conclusion}

This paper investigates and evaluates the performance of 1-bit ADCs with the 3-sigma and optimized uniform quantizer. The optimized quantizer brings significant BER performance improvement. Besides, the research suggests that one should use a large number of antennas to relieve the negative effect of the quantization distortion of 1-bit ADC, especially for a high coding rate. It is worth re-designing the protograph LDPC codes for this particular case of 1-bit ADCs for the massive MIMO coded communication systems.

\section{AcKNOWLEDGEMENT}

This research is funded by Vietnam National Foundation for Science and Technology Development (NAFOSTED) under grant number 102.04-2016.23
Hung N. Dang's research was supported by the Domestic Scholarship Programme of Vingroup Innovation Foundation under Grant number VINIF.2019.TS.30

\section{REFERENCES}

[1] D. C. Araújo, T. Maksymyuk, A. L. F. de Almeida, T. Maciel, J. C. M. Mota, and M. Jo, "Massive MIMO: survey and future research topics," IET Communications, vol. 10, no. 15, pp. 1938-1946, 2016.

[2] K. B. Letaief, W. Chen, Y. Shi, J. Zhang, and Y. A. Zhang, "The roadmap to 6G: AI empowered wireless networks," IEEE Communications Magazine, vol. 57, no. 8, pp. 84-90, Aug. 2019.

[3] Z. Zhang, Y. Xiao, Z. Ma, M. Xiao, Z. Ding, X. Lei, G. K. Karagiannidis, and P. Fan, "6G wireless networks: Vision, requirements, architecture, and key technologies," IEEE Vehicular Technology Magazine, vol. 14, no. 3, pp. 28-41, Sep. 2019.

[4] L. Fan, S. Jin, C. Wen, and H. Zhang, “Uplink achievable rate for massive MIMO systems with low-resolution ADC," IEEE Communications Letters, vol. 19, no. 12, pp. 2186-2189, Dec. 2015.

[5] W. Fukuda, T. Abiko, T. Nishimura, T. Ohgane, Y. Ogawa, Y. Ohwatari, and Y. Kishiyama, "Lowcomplexity detection based on belief propagation in a massive MIMO system," in Proceedings of the IEEE Vehicular Technology Conference (VTC), Jun. 2013, pp. 1-5.

[6] C. Zhang, Y. Jing, Y. Huang, and X. You, "Massive MIMO with ternary ADCs," IEEE Signal Processing Letters, vol. 27, pp. 271-275, 2020.

[7] T. Liu, J. Tong, Q. Guo, J. Xi, Y. Yu, and Z. Xiao, "Energy efficiency of massive MIMO systems with lowresolution adcs and successive interference cancellation," IEEE Transactions on Wireless Communications, vol. 18, no. 8, pp. 3987-4002, Aug. 2019.

[8] J. Dai, J. Liu, J. Wang, J. Zhao, C. Cheng, and J. Wang, "Achievable rates for full-duplex massive MIMO systems with low-resolution ADCs/DACs," IEEE Access, vol. 7, pp. 24343-24353, 2019.

[9] S. Gao, P. Dong, Z. Pan, and G. Y. Li, “Deep learning based channel estimation for massive MIMO with mixed-resolution ADCs," IEEE Communications Letters, vol. 23, no. 11, pp. 1989-1993, Nov. 2019.

[10] L. V. Nguyen, D. T. Ngo, N. H. Tran, A. L. Swindlehurst, and D. H. N. Nguyen, "Supervised and semi-supervised learning for MIMO blind detection with low-resolution ADCs," IEEE Transactions on Wireless Communications, vol. 19, no. 4, pp. 2427-2442, 2020.

[11] L. Xu, X. Lu, S. Jin, F. Gao, and Y. Zhu, “On the uplink achievable rate of massive MIMO system with lowresolution ADC and RF impairments," IEEE Communications Letters, vol. 23, no. 3, pp. 502-505, Mar. 2019.

[12] T. V. Nguyen, H. D. Vu, D. N. Nguyen, and H. T. Nguyen, "Performance analysis of protograph LDPC codes over large-scale MIMO channels with low-resolution ADCs," IEEE Access, vol. 7, pp. 145145-145160, 2019.

[13] Y. Cho and S. Hong, "One-bit successive-cancellation soft-output (OSS) detector for uplink MU-MIMO systems with one-bit ADCs," IEEE Access, vol. 7, pp. 2717227182, 2019.

[14] F. Mousavi and A. Tadaion, "A simple two-stage detector for massive MIMO systems with one-bit ADCs," in Proceedings of the 27th Iranian Conf. Electrical Engineering (ICEE), Apr. 2019, pp. 1674-1678.

[15] H. Dang, T. Nguyen, and H. Nguyen, "Improve uplink achievable rate for massive MIMO systems with lowresolution ADCs," in Proceedings of the 20th IEEE Conference on Communications and Electronics (ICCE), Phu Quoc, Vietnam, Jan. 2021.

[16] H. D. Vu, T. V. Nguyen, D. N. Nguyen, and H. T. Nguyen, 
"On design of protograph LDPC codes for large-scale MIMO systems," IEEE Access, vol. 8, pp. 46017-46029, 2020.

[17] D. Tse and P. Viswanath, Fundamentals Of Wireless Communication. Cambridge University Press, 2005.

[18] M. Srinivasan and S. Kalyani, "Analysis of massive MIMO with low-resolution ADC in Nakagami- $m$ fading," IEEE Communications Letters, vol. 23, no. 4, pp. 764767, Apr. 2019

[19] A. Gersho and R. M. Gray, Vector Quantization And Signal Compression. Kluwer Academic Publisher, 1992.

[20] Y. Xiong, N. Wei, and Z. Zhang, "A low-complexity iterative GAMP-based detection for massive MIMO with low-resolution ADCs," in Proceedings of the IEEE WCNC, Mar. 2017, pp. 1-6.

[21] D. Hui and D. L. Neuhoff, "Asymptotic analysis of optimal fixed-rate uniform scalar quantization," IEEE Transactions on Information Theory, vol. 47, no. 3, pp. 957977, Mar. 2001.

[22] T. Takahashi, S. Ibi, and S. Sampei, "On normalization of matched filter belief in gabp for large MIMO detection," in Proc. IEEE Vehicular Technology Conference (VTC), Sep. 2016, pp. 1-6.

[23] H. D. Vu, T. V. Nguyen, T. B. T. Do, and H. T. Nguyen, "Belief propagation detection for large-scale MIMO systems with low-resolution ADCs," in Proceedings of the International Conference on Advanced Technologies for Communications (ATC), 2019, pp. 68-73.

[24] S. ten Brink, G. Kramer, and A. Ashikhmin, "Design of low-density parity-check codes for modulation and detection," IEEE Transactions on Communications, vol. 52, no. 4, pp. 670-678, Apr. 2004.

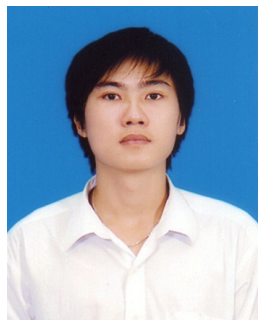

Hung N. Dang received the B.Sc. and M.Sc. degrees in information technology from Posts and Telecommunications Institute of Technology (PTIT), Hanoi, Vietnam. He is currently a lecturer of Faculty of Information Technology, Posts and Telecommunications Institute of Technology (PTIT), Hanoi, Vietnam. His research interests lie in Massive MIMO communications, channel coding design and analysis, wireless sensor networks.

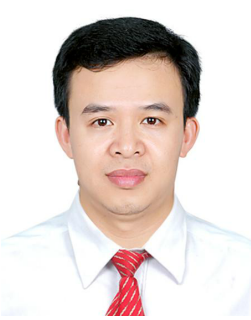

Thuy V. Nguyen received the B.Sc., M.Sc. and $\mathrm{Ph} . \mathrm{D}$. degrees in electrical engineering from Hanoi University of Science and Technology (HUST), Hanoi, Vietnam, New Mexico State University, Las Cruces, NM, USA, and the University of Texas at Dallas, Richardson, TX, USA, respectively. He is currently a lecturer of Faculty of Information Technology, Posts and Telecommunications Institute of Technology (PTIT), Hanoi, Vietnam. Before joining PTIT, he was a Member of Technical Staff with Flash Channel Architecture, Seagate, Fremont, CA, USA. His research interests lie in the areas of coding theory and its applications in nextgeneration communication systems.

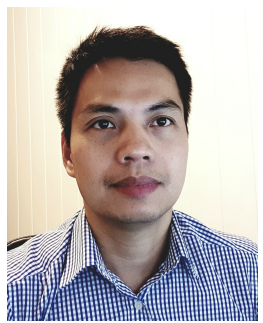

Hieu T. Nguyen received the B.Sc., M.Sc. and $\mathrm{Ph} . \mathrm{D}$. degrees in electrical engineering from Hanoi University of Science and Technology, University of Saskatchewan, Canada and Norwegian University of Science and Technology, respectively. He is a faculty member of Faculty of Technology, Natural Sciences, and Maritime Sciences, University of South-Eastern Norway (USN). 